\title{
Combined reconstruction of the medial patellotibial and patellofemoral ligaments
}

\author{
N. Maffulli1,2,3, R. Aicale ${ }^{1}$, D. Tarantino ${ }^{1}$, D. A. Young ${ }^{4}$ \\ 1 Department of Musculoskeletal Disorders, School of Medicine and Surgery, University of Salerno, Salerno, Italy. \\ 2 Queen Mary University of London, Barts and the London School of Medicine and Dentistry, Centre for Sports \\ and Exercise Medicine, Mile End Hospital, 275 Bancroft Road, London E1 4DG, England \\ 3 Institute of Science and Technology in Medicine, Keele University School of Medicine, Stoke on Trent, England \\ 4 Melbourne Orthopaedic Group, Windsor, Victoria, Australia
}

\section{CORRESPONDING AUTHOR:}

Rocco Aicale

Department of Musculoskeletal Disorders, School of Medicine and Surgery,

University of Salerno,

Salerno, Italy

E-mail: aicale17@gmail.com

Phone: 3458485495

DOI:

10.32098/mltj.02.2019.06

LEVEL OF EVIDENCE:

5

\begin{abstract}
SUMMARY
Background. Patellofemoral instability is commonly associated with the rupture of the medial patellofemoral ligament (MPFL), and the role of the medial patellotibial ligament (MPTL) is slowly emerging. Soft tissue reconstructions are able to restore patellar stability.

Methods. We present a surgical technique for the combined reconstruction of the MPFL and MPTL using gracilis or semitendinosus tendon (ST) autograft and two patellar tunnels, looping the free end of the tendon graft around the adductor magnus tendon.

Results. In this surgical technique the femoral physeal plate is not affected, no femoral tunnel needs to be drilled, no hardware is needed to fix the graft to the bone. Conclusion. The technique described is simple, safe, inexpensive, reproducible and does not require intraoperative fluoroscopy.
\end{abstract}

\section{KEY WORDS}

Patellar instability; patellar dislocation; hamstring tendons; gracilis tendon; semitendinosus tendon; reconstruction

\section{INTRODUCTION}

Patellofemoral instability is frequent (1), and it is commonly associated with pain, reduced quality of life, decreased activity and long-term osteoarthritis (2). In children and adolescents (10-17 years), patellofemoral joint injury occurs in $29-43 / 100.000$, and is 10 times more frequent than in adults (2.3-7.0/100.000) (3). The medial patellofemoral ligament (MPFL) is the main restrictor to the lateral translation of the patella (4). Important contributors to such restriction are the medial patellotibial ligament (MPTL) and the medial patellomeniscal ligament (MPML) (4), which contribute to the limitation of patella dislocation above $30^{\circ}$ of knee flexion. The MPTL contributes from $26 \%$ to $46 \%$ to limit patella dislocation when the limb is in extension or at $90^{\circ}$ of flexion, respectively (4).
The MPFL is located in the second layer of the anteromedial aspect of the knee (5), and its femoral origin lies between the adductor tubercle and the medial epicondyle (6). From this narrow femoral origin, the MPFL widens to an insertion occupying approximately half of the proximal medial patella (7-9). The MPFL is the primary passive restraint in lateral patellar translation, tearing when the patella dislocates laterally (10). MPFL reconstruction effectively stabilises the patella, and has become one of the most widely used surgical techniques for the management of chronic lateral patellar instability (11).

The MPTL influences tilt and rotation movements of the knee: combined reconstruction of both MPTL and MPFL may contribute to maintain the normal kinematics of the patellofemoral joint throughout the range of motion (12). 
Several techniques have been described (13) to control patellar instability. Many of these are employed in patients with skeletal immaturity, high riding patella, trochlear dysplasia, and ligament hyperlaxity (14). They vary in terms of graft choice, type of fixation, patellar and femoral attachments and graft tension at the time of fixation $(13,15-17)$. A standard surgical technique to manage this condition remains elusive (15).

We present a simple and reproducible method for combined MPTL and MPFL reconstruction that is not based on radiographic landmarks and can be performed in skeletally immature patients.

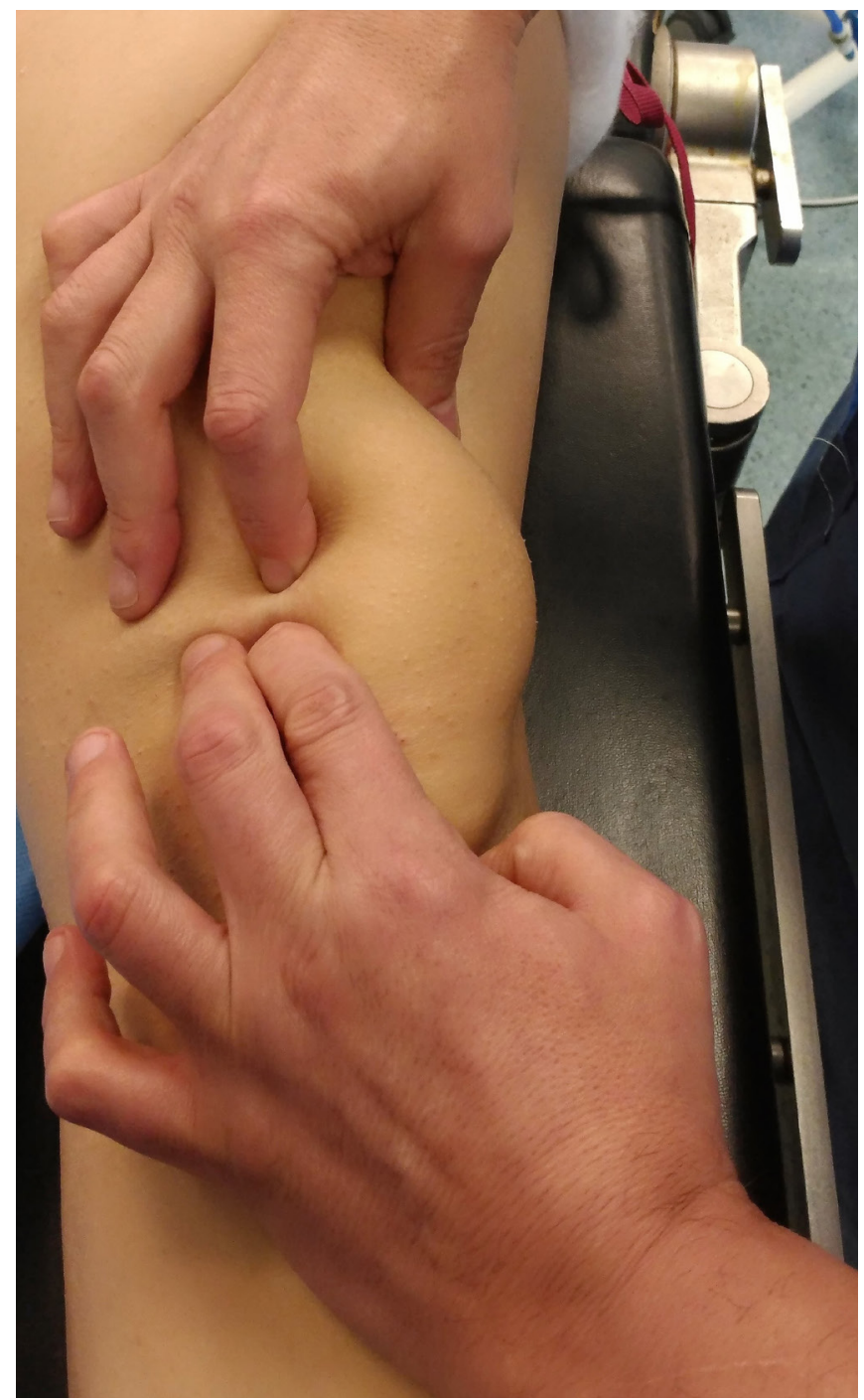

Figure 1. Instability is evaluated on the operating table, diagnosis is confirmed by a horizontal lateral displacement of the patella of at least two quarters of its transverse diameter.

\section{SURGICAL TECHNIQUE}

The combined MPFL and MPTL reconstruction is performed in patients with objective recurrent patellar instability in whom conservative management failed. Instability is evaluated under anesthesia on the operating table, and the diagnosis is confirmed by an abnormal horizontal lateral movement of the patella for more than 2 quarters of its diameter, with a gentle force applied on its medial aspect with the knee fully extended and/or at $30^{\circ}$ flexion (Figure 1).

With the patient supine and a thigh tourniquet, a diagnostic arthroscopy is performed to address and document any

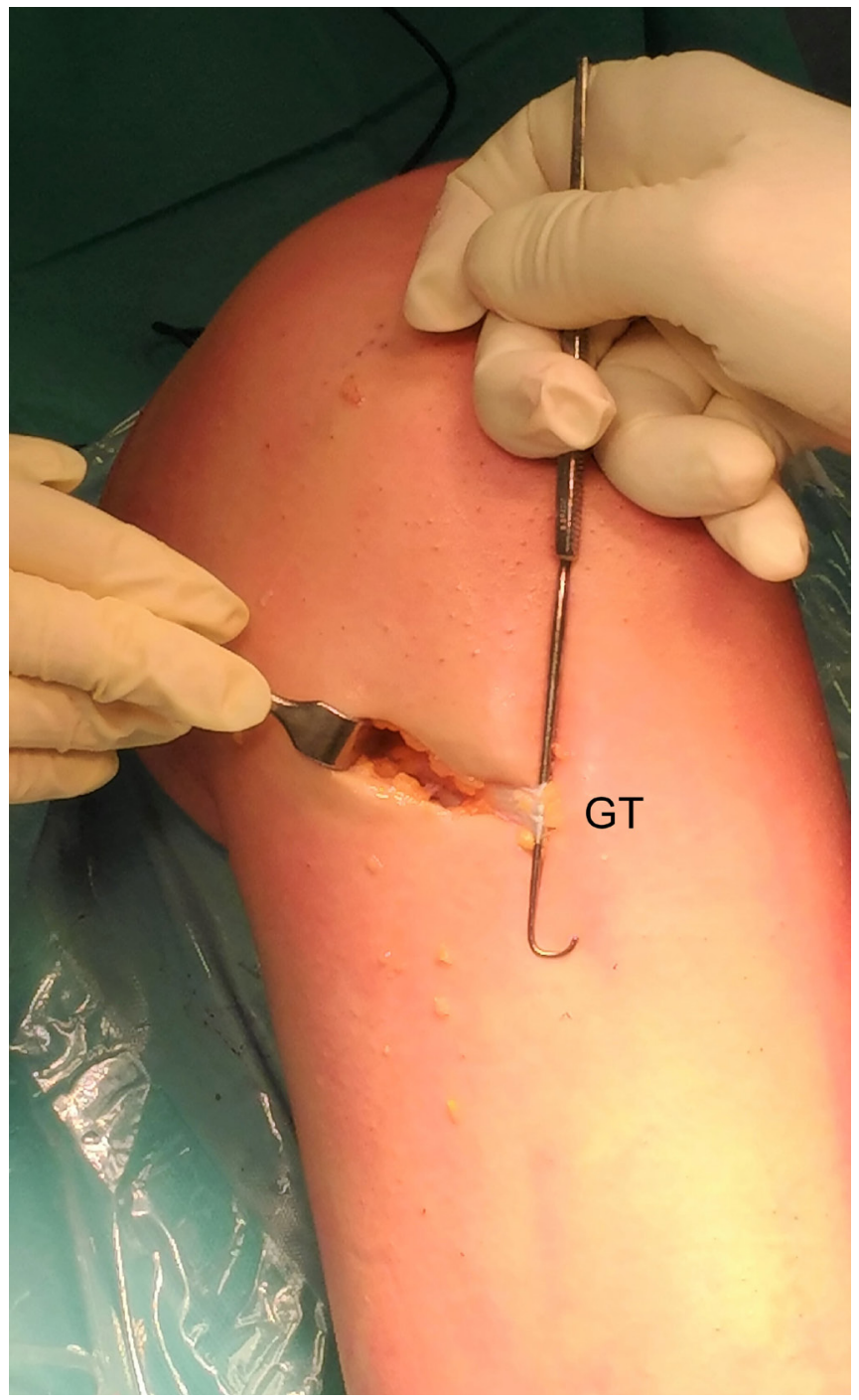

Figure 2. GT: gracilis tendon; After a vertical skin incision of $2-2.5 \mathrm{~cm}$ over the pes anserinus, the gracilis tendon was palpate and exposed. 
intra-articular lesions, particularly osteochondral abnormalities of the patellofemoral joint or evidence of patellar maltracking.

\section{Gracilis Tendon Harvest}

A vertical 2-2.5 $\mathrm{cm}$ skin incision is used to expose the pes anserinus. The anserinus bursa is incised horizontally and opened in the line of the tendon, and the gracilis (proximal) and semitendinosus (distal) tendons are identified and separated. The gracilis tendon is palpated, exposed and released with a tendon stripper (Figure 2-3). It is important not to go deeper to prevent any injury to the underlying superficial medial collateral ligament (MCL). After harvesting the tendon, whipstitch sutures are used to preparing proximal end using strong absorbable sutures (No. 2 Vicryl, Ethicon, Somerville, New Jersey).

\section{Patellar Exposure and Tunnels}

The patella is then prepared using a vertical incision approximately $3 \mathrm{~cm}$ long on the medial aspect of the patella itself. Two parallel tunnels are prepared, one in the proximal half of the patella and the other in the distal half of it, initial-

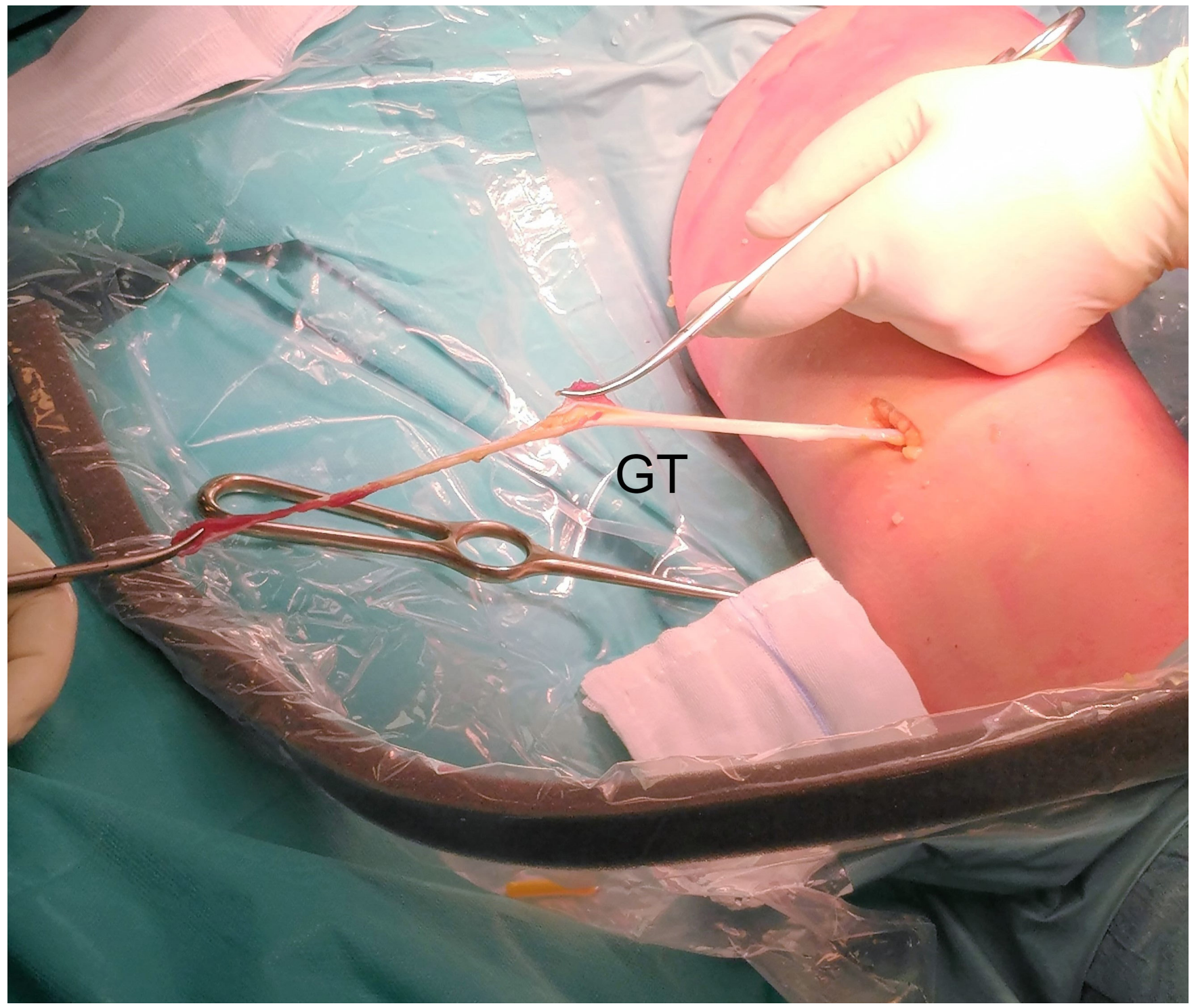

Figure 3. GT: gracilis tendon; Gracilis tendon is harvested with a tendon stripper. 
ly with 2 guidewires, followed by a cannulated $5 \mathrm{~mm}$ drill (Figure 4). The entry points of each tunnels are prepared in the medial patella border spaced by an interval at least $20 \mathrm{~mm}$, checked using a ruler, while the exit points of each tunnels are on the anterior aspect of the patella $1.5-2 \mathrm{~cm}$ from its medial border. This allow to the MPFL and MPTL reconstruction to occupy a total of approximately $20 \mathrm{~mm}$, similar to the reported mean width of $22 \mathrm{~mm}$ of the intact ligaments on the medial patella (8). (Figure 5)

\section{Adductor Tubercle Exposure}

The adductor tubercle and the medial epicondyle are palpated, and this location confirmed visually. Another 2 $\mathrm{cm}$ skin incision is made along the adductor magnus (AM) tendon, $2.5 \mathrm{~cm}$ proximal to the medial femoral epicondyle (Figure 6). The approach is made in line with the medial intermuscular septum, and the AM tendon is easily identified by finger palpation. Anatomically, the AM tendon sits flush to the posteromedial aspect of the femur and attaches to the adductor tubercle just proximal to the medial epicondyle. Once identified, the AM tendon and its hiatus are dissected as distally as possible to the anatomic femoral attachment of the graft to the original attachment point of the MPFL.

\section{Graft Passage and Suture}

The free end of the tendon graft is passed through the patellar tunnels and then through the second layer of the knee, remaining extra-articular. Finally, the free end of the tendon graft is looped around the AM tendon and then back to itself towards the patella (Figure 7). Therefore, the AM hiatus is used as an elastic pulley for the graft which is secured to AM by Vicryl (Ethicon, Somerville, New Jersey) with $30^{\circ}$ of knee flexion. The knee is then cycled several times through the full range of motion, and the graft tension is established on the basis of the possible manual lateralization of the patella, about $10 \mathrm{~mm}$ or one quadrant, to avoid over constraint. The wounds are closed in layers, and routine dressings and bandages are applied.

\section{Rehabilitation Protocol}

Partial weight-bearing as tolerated is allowed immediately after surgery, and no brace is used. As soon as the effect of the anaesthesia is over, patients are encouraged to perform simple exercises such as moving the toes, ankle, knee and hip of the operated leg. Isometric strengthening exercises began once pain is controlled. Active mobilization is encouraged after 4 weeks, stretching exercises are allowed.
After 8 weeks, gentle jogging on the-spot on a trampoline is started. (Table I)

\section{DISCUSSION}

We present a simple technique for the combined MPFL and MPTL reconstruction in skeletally immature patients, ensuring reconstruction and stabilization of the patella with no other associated procedures, such as trochleoplasty or medialization of the anterior tibial tuberosity through osteotomy. The gracilis tendon is used as graft because of its length, making it possible to preserve its tibial insertion (14).

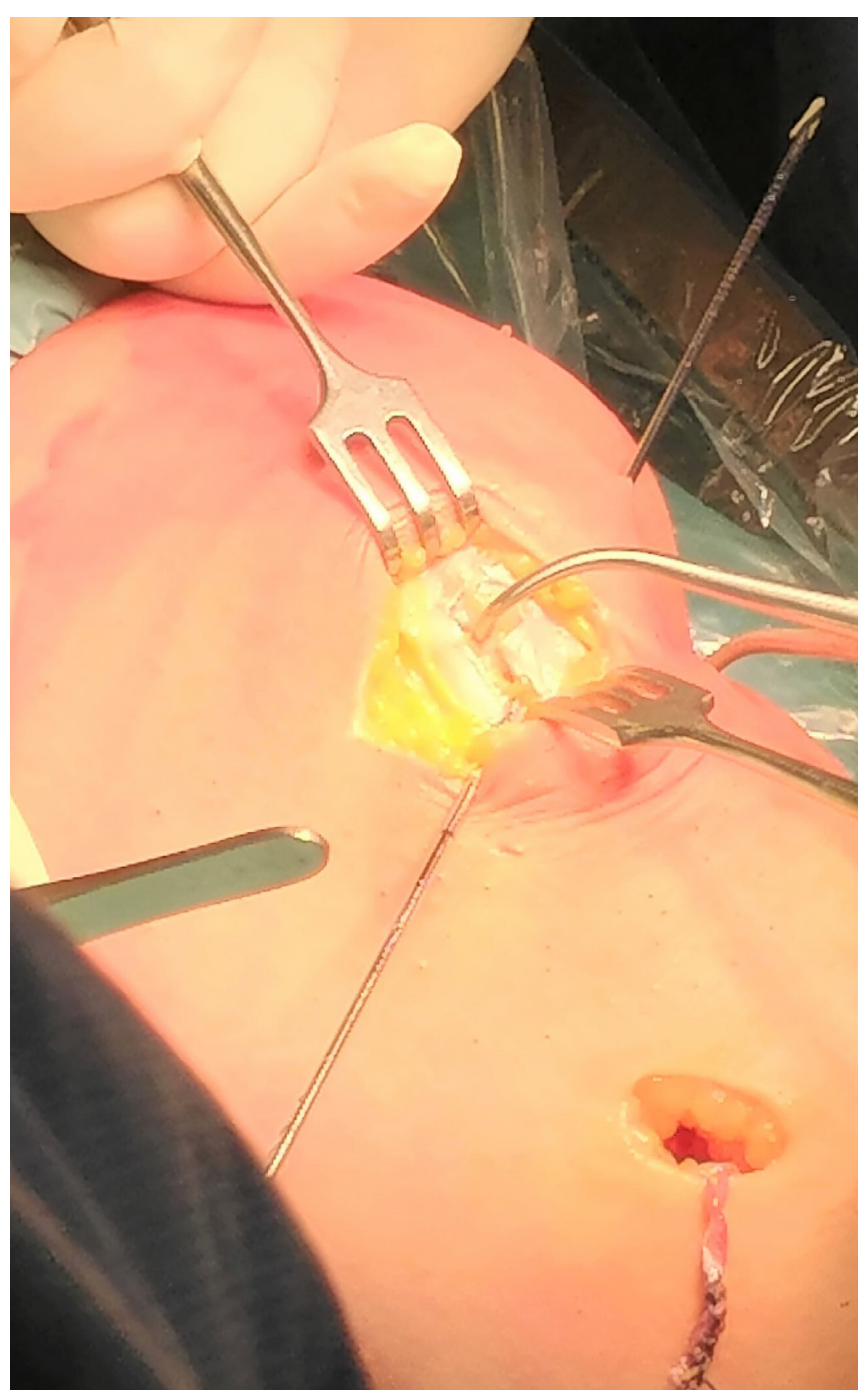

Figure 4. Two tunnel are prepared, in the distal half of patella, initially with 2 guidewires, followed by a cannulated $5-\mathrm{mm}$ drill. 


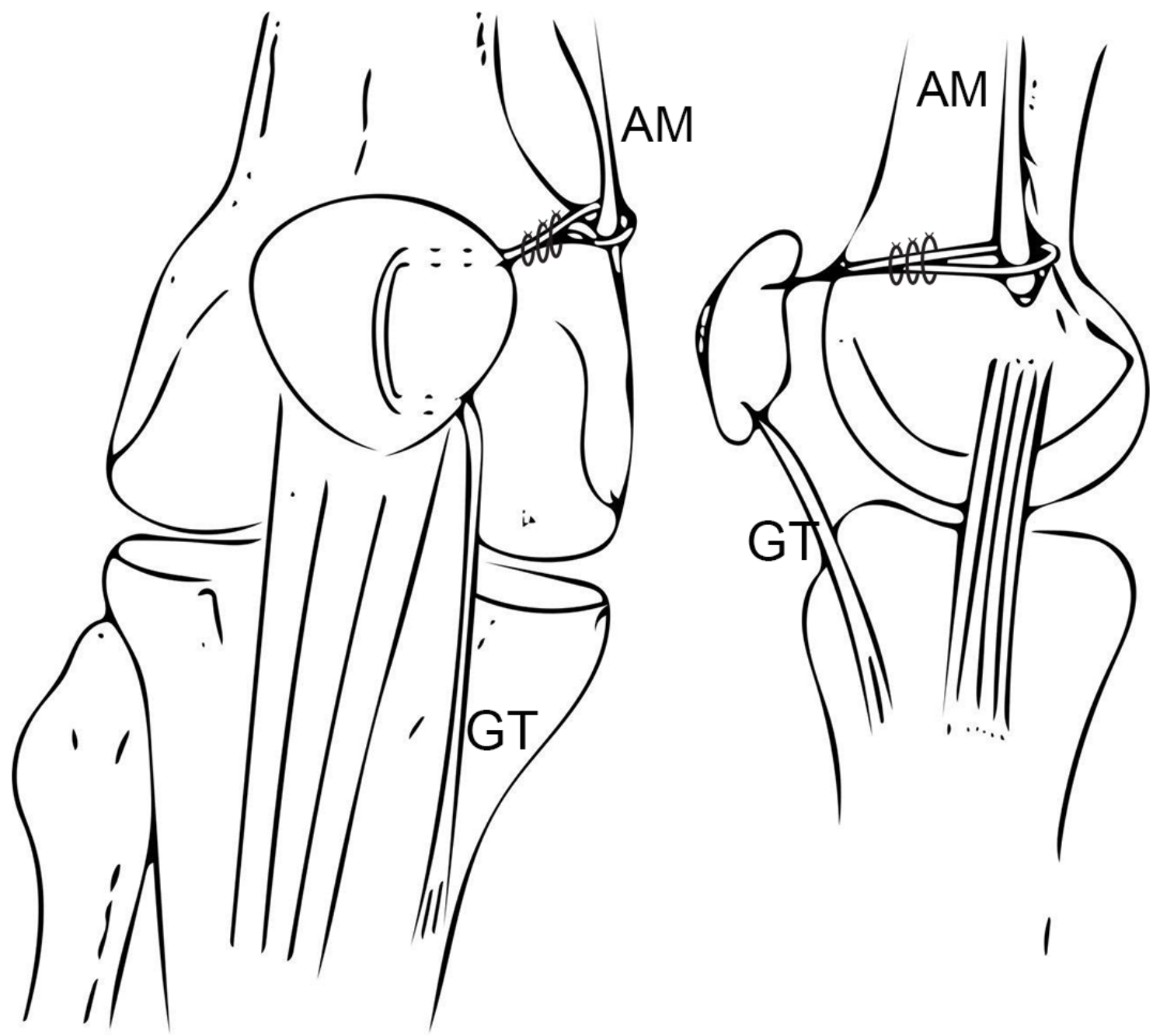

Figure 5. GT: gracilis tendon, AM: adductor magnus tendon; Schematic representation of the patella tunnels in anteroposterior and inferosuperior view.

Table I. AM: Adductor Magnus. Step-by Step Medial Patellofemoral Ligament and Medial Patellotibial Reconstruction Technique.

\begin{tabular}{cl}
\hline Phase & Description \\
\hline 1 & $\begin{array}{l}\text { Instability is evaluated under anesthesia and a diagnostic arthroscopy is performed to address and document any intra- } \\
\text { articular lesions }\end{array}$ \\
\hline 2 & $\begin{array}{l}\text { A vertical skin incision of } 2-2.5 \mathrm{~cm} \text { is used to approach the gracilis tendon which is palpated, exposed and released with a } \\
\text { tendon stripper }\end{array}$ \\
\hline 3 & Whipstitch sutures are passed to the ends of the tendon using strong absorbable sutures. \\
\hline 5 & $\begin{array}{l}\text { A vertical incision approximately } 3 \mathrm{~cm} \text { long on the medial aspect of the patella is made and two parallel tunnels are prepared } \\
\text { spaced by } 20 \mathrm{~mm} \text {, the exit points are situated on the anterior aspect of the patella at } 2 \mathrm{~cm} \text { from its medial margin }\end{array}$ \\
\hline 6 & The graft is passed through the patellar tunnel, looped around the AM tendon and back to patella itself, secured by Vicryl \\
\hline 7 & The wound is closed in layers, and routine dressings and bandages are applied \\
\hline
\end{tabular}




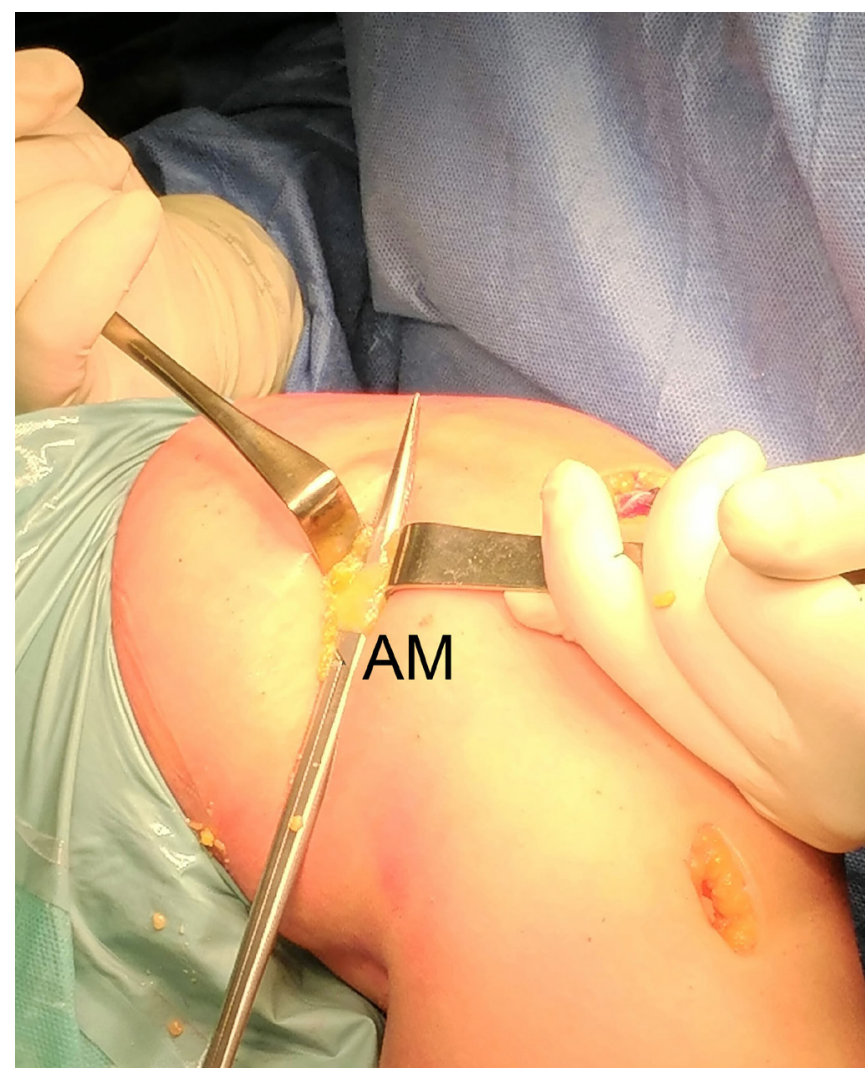

Figure 6. AM: adductor magnus tendon; The adductor magnus tendon is identified at $2.5 \mathrm{~cm}$ proximal to the medial femoral epicondyle.

The procedure presented is performed with relatively small incisions, and this makes the technique appealing in both female and young patients. Moreover, patellar stabilization can be achieved with minimal exposure of soft tissues.

Despite the good results generally obtained with MPFL reconstruction to control lateral patellar instability, some aspects remain still controversial. Failure of MPFL reconstruction may occur because of incorrect surgical indication, inappropriate surgical technique and/or patient selection, but several biomechanical studies demonstrated that femoral tunnel position is the most important factor affecting the biomechanical behaviour of the MPFL graft $(16,18)$.

Malposition of the femoral tunnel may change the isometry of the graft, increasing patellofemoral contact pressures (19). Conversely, a non-anatomic femoral attachment to the adductor tubercle did not alter pressures on the patellofemoral joint, as demonstrated by a biomechanical study using cadaver is knees (20). These nonanatomic reconstructions may exhibit a realistic physiometric behaviour which

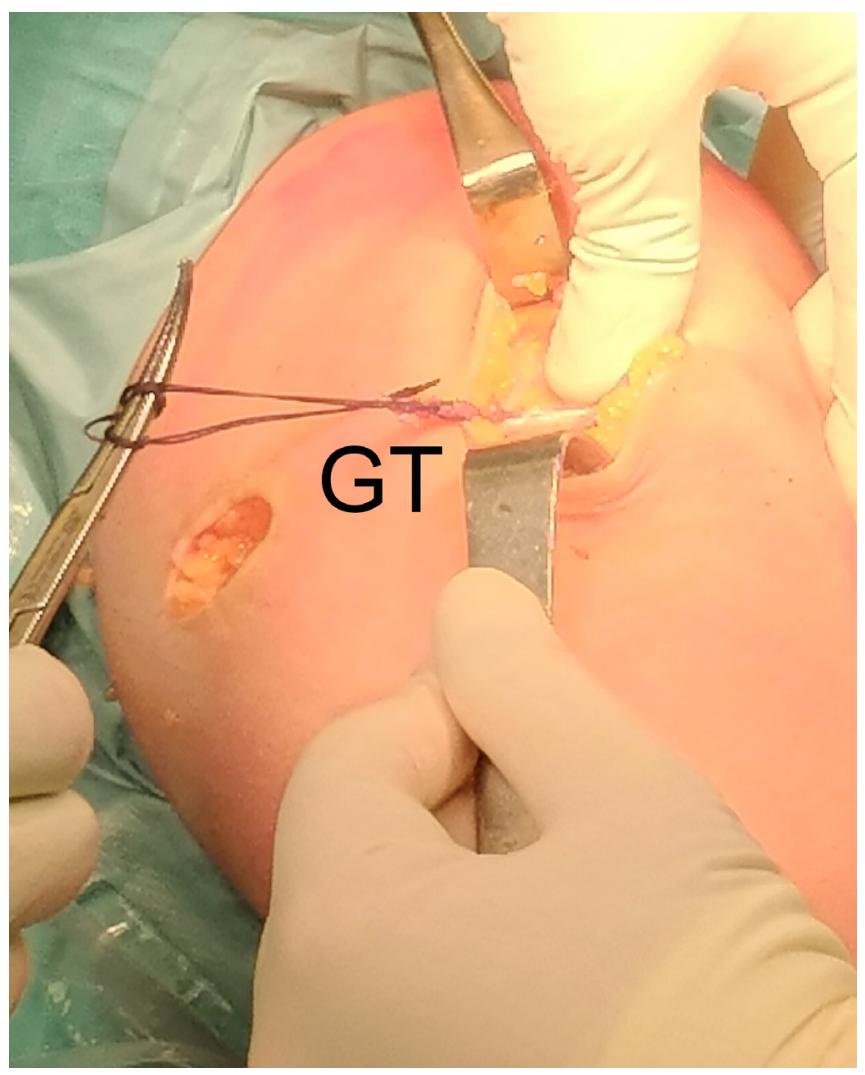

Figure 7. GT: gracilis tendon. The free end of the gracilis tendon graft is looped around the adductor magnus tendon and then back to itself towards the patella.

prevents overpressures of the patellofemoral joint (21), with minimal changes in MPFL length during knee flexion from full extension to $90^{\circ}$, which is only $1.1 \mathrm{~mm}$ (22).

No clinical signs of patellofemoral overload, recurrent patellar instability or radiologic osteoarthritis using the AM tendon as femoral attachment were observed in the short term (16). Furthermore, although this method is not completely anatomic (normally, the MPFL inserts 5 to 10 $\mathrm{mm}$ distally to the AM), the elastic nature of this attachment might compensate any small length mismatch (23).

Regarding the graft, some authors encouraged the use of the gracilis to reconstruct the MPFL for its documented resistance to tensile load (24). The native MPFL have a mean tensile strength of $208 \mathrm{~N}$ (7). The mean maximum load for 1 strand of gracilis was $837 \pm 138 \mathrm{~N}$ and $160 \pm 44 \mathrm{~N}$ per millimetre of stiffness, while 2 strands of the same tendon had approximately $1550 \pm 428 \mathrm{~N}$ of strength and $336 \pm 141$ $\mathrm{N}$ per millimetre of stiffness (24). The function of MPFL and MPTL can be duplicated by the gracilis tendon, which is long and strong enough. 
The semitendinosus (ST) tendon can be used with the same procedure to restore patellar stability. A biomechanical study demonstrated that the mean maximum load for 1 strand of a ST was $1060 \pm 227 \mathrm{~N}$ and $213 \pm 44 \mathrm{~N}$ per millimetre of stiffness, while 2 strands had $2330 \pm 452 \mathrm{~N}$ of strength and $469 \pm 185 \mathrm{~N}$ per millimetre of stiffness (24). The tendon of ST is long enough but markedly stronger than the gracilis.

The surgical technique described in this article is simple, safe, inexpensive, and reproducible. The femoral physeal plate is not affected, no femoral tunnel needs to be drilled, no hardware is needed to fix the graft to the bone, and it does not require intraoperative fluoroscopy (Table II).

\section{CONCLUSIONS}

In conclusion, this technique is easy to perform, safe, inexpensive and an adequate treatment for chronic patellar instability.

\section{REFERENCES}

1. Andrish J. Surgical Options for Patellar Stabilization in the Skeletally Immature Patient. Sports Med Arthrosc Rev. 2017;25(2):100-4.

2. Fithian DC, Paxton EW, Stone ML, Silva P, Davis DK, Elias $\mathrm{DA}$, et al. Epidemiology and natural history of acute patellar dislocation. Am J Sports Med. 2004;32(5):1114-21.

3. Hoetzel J, Preiss A, Heitmann MA, Frosch K-H. Knee injuries in children and adolescents. Eur J Trauma Emerg Surg Off Publ Eur Trauma Soc. 2014;40(1):23-36.

4. Philippot R, Boyer B, Testa R, Farizon F, Moyen B. The role of the medial ligamentous structures on patellar tracking during knee flexion. Knee Surg Sports Traumatol Arthrosc Off J ESSKA. 2012;20(2):331-6.

5. Warren LF, Marshall JL. The supporting structures and layers on the medial side of the knee: an anatomical analysis. J Bone Joint Surg Am. 1979;61(1):56-62.

6. Nomura E, Inoue M, Osada N. Anatomical analysis of the medial patellofemoral ligament of the knee, especially the femoral attachment. Knee Surg Sports Traumatol Arthrosc Off J ESSKA. 2005;13(7):510-5.

7. Amis AA, Firer P, Mountney J, Senavongse W, Thomas NP. Anatomy and biomechanics of the medial patellofemoral ligament. The Knee. 2003;10(3):215-20.

8. Kang HJ, Wang F, Chen BC, Su YL, Zhang ZC, Yan CB. Functional bundles of the medial patellofemoral ligament. Knee Surg Sports Traumatol Arthrosc Off J ESSKA. 2010;18(11):1511-6.

9. Reider B, Marshall JL, Koslin B, Ring B, Girgis FG. The anterior aspect of the knee joint. J Bone Joint Surg Am. 1981;63(3):351-6.

10. Zaffagnini S, Dejour D, Grassi A, Bonanzinga T, Marcheggiani M, Colle F, et al. Patellofemoral anatomy and biomechanics: Current concepts. Joints. 2013;1(2):15-20.
Table II. List of Advantages and Limitations

\begin{tabular}{l} 
Advantages \\
\hline Simple, safe, reproducible \\
\hline $\begin{array}{l}\text { Adequate for the treatment of recurrent patellar instability in } \\
\text { children and adolescents }\end{array}$ \\
\hline No intraoperative fluoroscopy \\
\hline No interference with subsequent MRI imaging \\
\hline Kinematics of the patellofemoral joint is respected \\
\hline Limitations \\
\hline Risk of patellar bone bridge fracture \\
\hline
\end{tabular}

\section{Conflict of Interest}

The authors declare that they have no conflict of interest (25)

11. Neri T, Philippot R, Carnesecchi O, Boyer B, Farizon F. Medial patellofemoral ligament reconstruction: Clinical and radiographic results in a series of 90 cases. Orthop Traumatol Surg Res. 2015;101(1):65-9.

12. Hinckel BB, Gobbi RG, Bonadio MB, Demange MK, Pécora JR, Camanho GL. Reconstruction of medial patellofemoral ligament using quadriceps tendon combined with reconstruction of medial patellotibial ligament using patellar tendon: initial experience. Rev Bras Ortop. 2016;51(1):75-82.

13. Becher C, Kley K, Lobenhoffer P, Ezechieli M, Smith T, Ostermeier S. Dynamic versus static reconstruction of the medial patellofemoral ligament for recurrent lateral patellar dislocation. Knee Surg Sports Traumatol Arthrosc Off J ESSKA. 2014;22(10):2452-7.

14. Giordano M, Falciglia F, Aulisa AG, Guzzanti V. Patellar dislocation in skeletally immature patients: semitendinosous and gracilis augmentation for combined medial patellofemoral and medial patellotibial ligament reconstruction. Knee Surg Sports Traumatol Arthrosc Off J ESSKA. 2012;20(8):1594-8.

15. Haupert A, Lorbach O. Anatomic reconstruction of the medial patellofemoral ligament using the fascia lata as an autograft. Arthrosc Tech. 2015;4(1):e57-63.

16. Monllau JC, Masferrer-Pino À, Ginovart G, Pérez-Prieto D, Gelber PE, Sanchis-Alfonso V. Clinical and radiological outcomes after a quasi-anatomical reconstruction of medial patellofemoral ligament with gracilis tendon autograft. Knee Surg Sports Traumatol Arthrosc. 2017;25(8):2453-9.

17. Schöttle PB, Fucentese SF, Romero J. Clinical and radiological outcome of medial patellofemoral ligament reconstruction with a semitendinosus autograft for patella instability. Knee Surg Sports Traumatol Arthrosc. 2005;13(7):516-21.

18. Sanchis-Alfonso V, Ramirez-Fuentes C, Montesinos-Berry E, Domenech J, Martí-Bonmatí L. Femoral insertion site of the 
graft used to replace the medial patellofemoral ligament influences the ligament dynamic changes during knee flexion and the clinical outcome. Knee Surg Sports Traumatol Arthrosc Off J ESSKA. 2017;25(8):2433-41.

19. Stephen JM, Kittl C, Williams A, Zaffagnini S, Marcheggiani Muccioli GM, Fink C, et al. Effect of Medial Patellofemoral Ligament Reconstruction Method on Patellofemoral Contact Pressures and Kinematics. Am J Sports Med. 2016;44(5):1186-94.

20. Melegari TM, Parks BG, Matthews LS. Patellofemoral contact area and pressure after medial patellofemoral ligament reconstruction. Am J Sports Med. 2008;36(4):747-52.

21. Panagopoulos A, van Niekerk L, Triantafillopoulos IK. MPFL reconstruction for recurrent patella dislocation: a new surgical technique and review of the literature. Int J Sports Med. 2008;29(5):359-65.
22. Steensen RN, Dopirak RM, McDonald WG. The anatomy and isometry of the medial patellofemoral ligament: implications for reconstruction. Am J Sports Med. 2004;32(6):1509-13.

23. Monllau JC, Pelfort X, Gelber P, Tey M. Combined tibial tubercle realignment and MPFL reconstruction. In: Patellar instability surgery in clinical practice. Springer; 2013. pag. 39-45.

24. Hamner DL, Brown CH, Steiner ME, Hecker AT, Hayes WC. Hamstring tendon grafts for reconstruction of the anterior cruciate ligament: biomechanical evaluation of the use of multiple strands and tensioning techniques. J Bone Joint Surg Am. 1999;81(4):549-57.

25. Padulo J., Oliva F., Frizziero A., Maffulli N. Muscles, Ligaments and Tendons Journal - Basic principles and recommendations in clinical and field Science Research: 2018 update. MLTJ 2018; 8(3): $305-307$ 